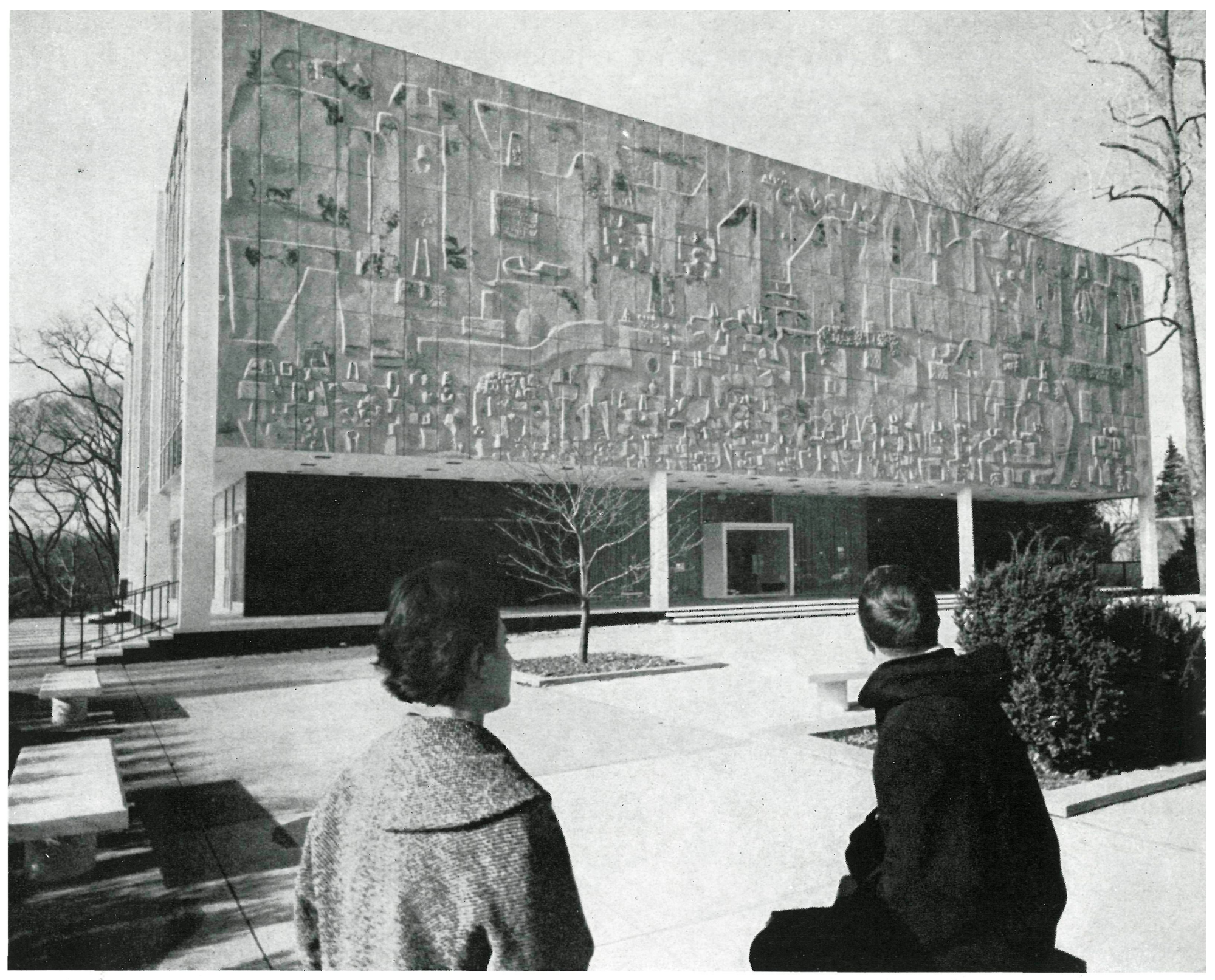

\title{
edificio para oficinas
}

Este edificio ha obtenido el primer premio del Instituto Americano de Arquitectos, en 1960, y el Certificado de Mérito para un proyecto destacado, en 1959, de la Asociación de Arquitectos de Nueva York.

\section{en Hartford - U.S.A.}

SHERWOOD, MILLS y SMITH, arquitectos

$131 \cdot 15$

Se ha dedicado un cuidado especial en buscar el emplazamiento adecuado y óptimo para este edificio, con vistas a crear para él un pequeño oasis de tranquilidad en medio de las concurridas y trafagosas calles del centro urbano. Se levanta, pues, en un parque frondoso, rodeado curridas y trafagosas calles del centro urbano. Se levanta, pues, en un parque frondoso, rodeado
de olmos y hayas centenarios, y de alamedas y praderas verdes. Una terraza elevada, frente al edificio, invita a la reunión, al descanso y a la observación deleitable de los bellos paisajes circundantes.

El programa se desarrolla en tres plantas dedicadas a oficinas varias, despachos y salas de consejos. 


\section{fachada norte y cafetería para empleados}

En todas ellas se observa la misma disposición: oficinas, zonas de descanso, recepciones, vestíbulos y partes más notables, en el perímetro del edificio, envolviendo a un núcleo central de servicios. Fácilmente puede apreciarse la efectividad de esta planimetría y su perfecto funcionamiento.

Todo el bloque está rodeado, en su planta baja, por una magnífica galeríaporche que alcanza mayores dimensiones en la fachada principal, por su carácter más noble y aparente; en la parte posterior se dispone una zona para ensanche de la cafetería para empleados, con pavimento de losas de piedra y sombrillas coloreadas acopladas a las mesas de tertulia.

\section{Exteriores:}

Lo más espectacular del edificio consiste, sin duda, en el gran bajorrelieve que ocupa la parte alta de la fachada principal, realizado por el prestigioso escultor Costantino Nivola. Ocupa una extensión de $33,5 \times 9,15 \mathrm{~m}$ de fachada y es, sin duda, uno de los mayores de esta clase en el país.
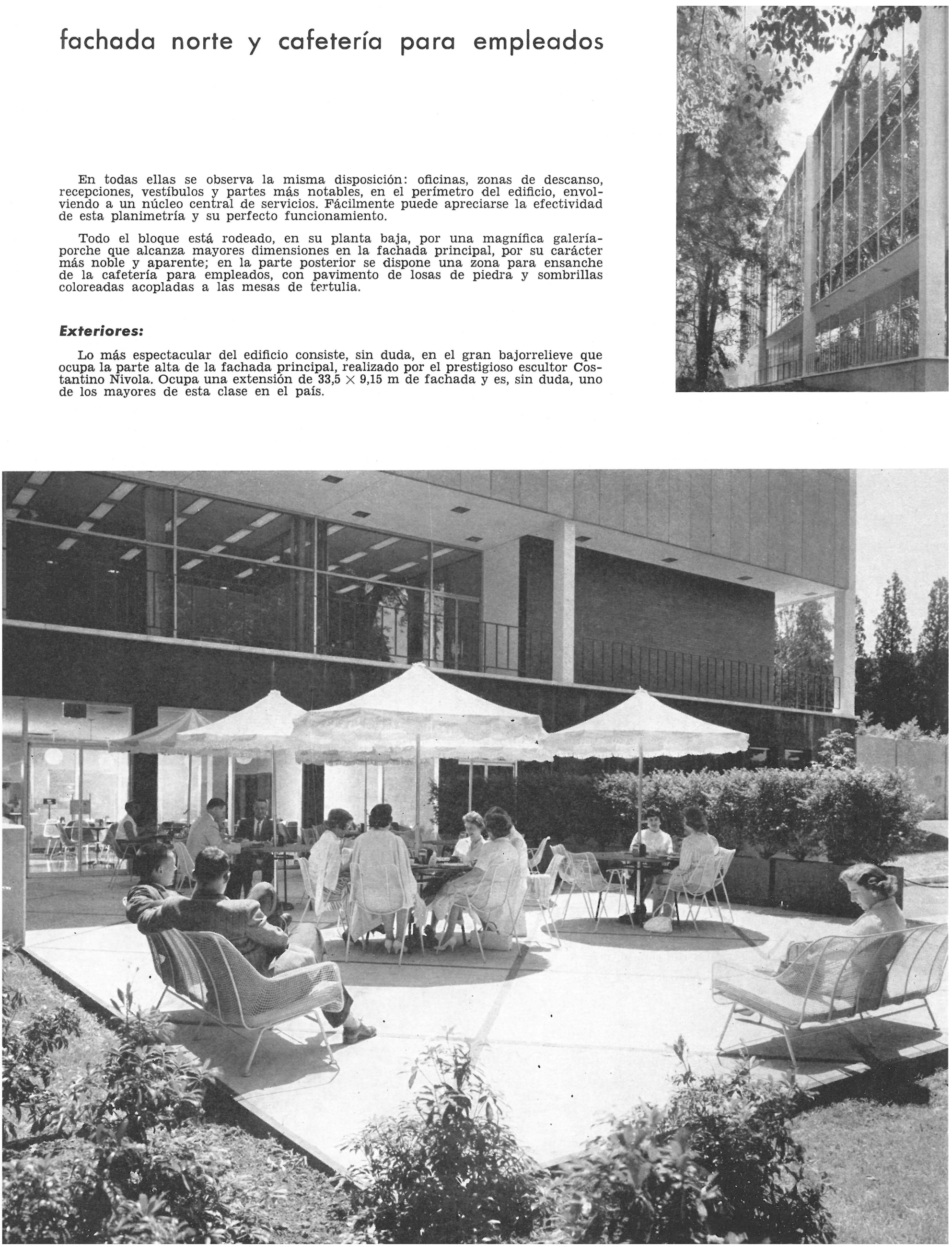
vestíbulo

1. Recepción.

2. Sala de Consejos y oficina del Presidente.

3. Secretaría y oficinas.

4. Servicios.

5. Máquinas.

6. Marina interior.

7. Vestíbulo de empleados.

8. Servicio de agencias.
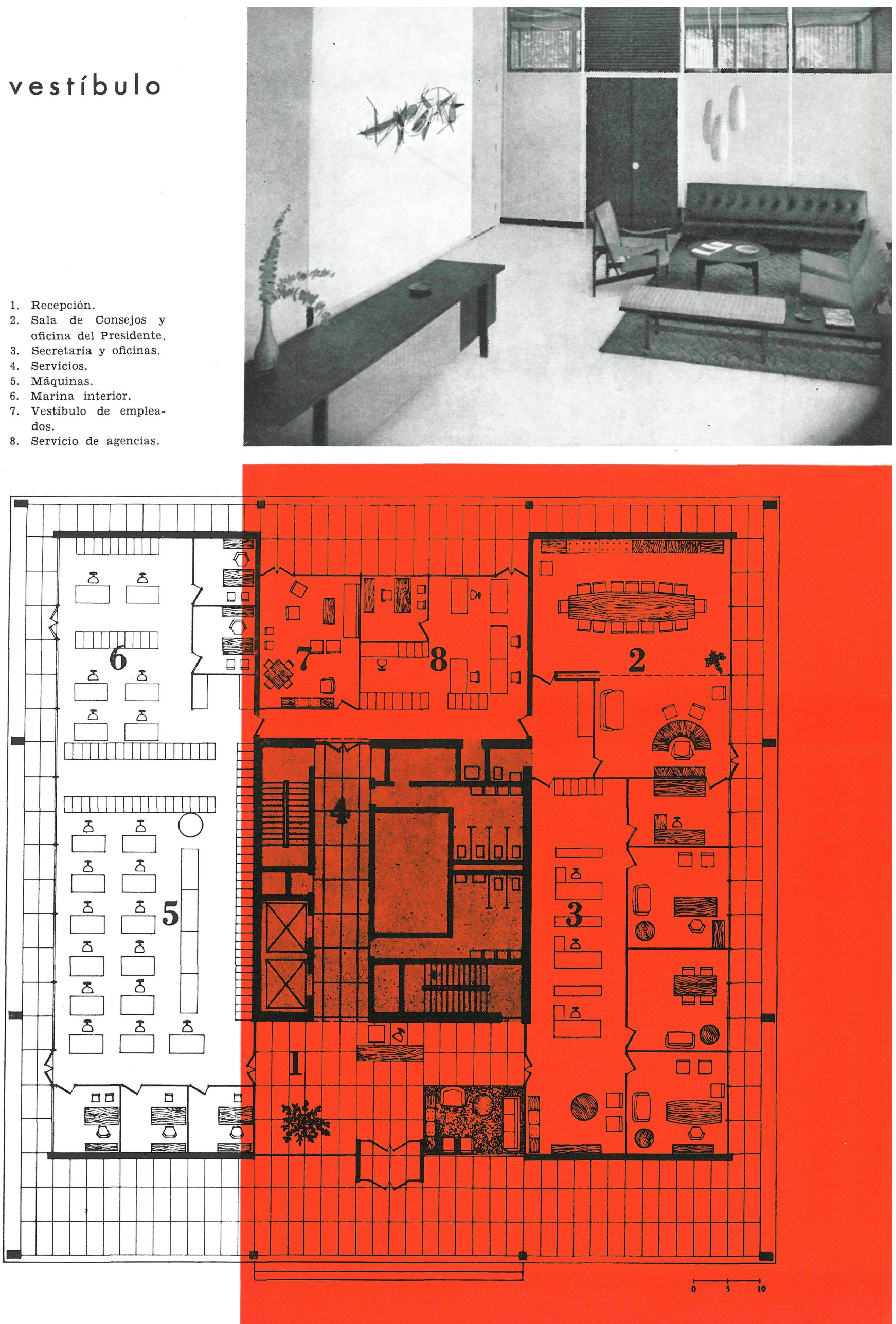


\section{sala de consejo y despacho}

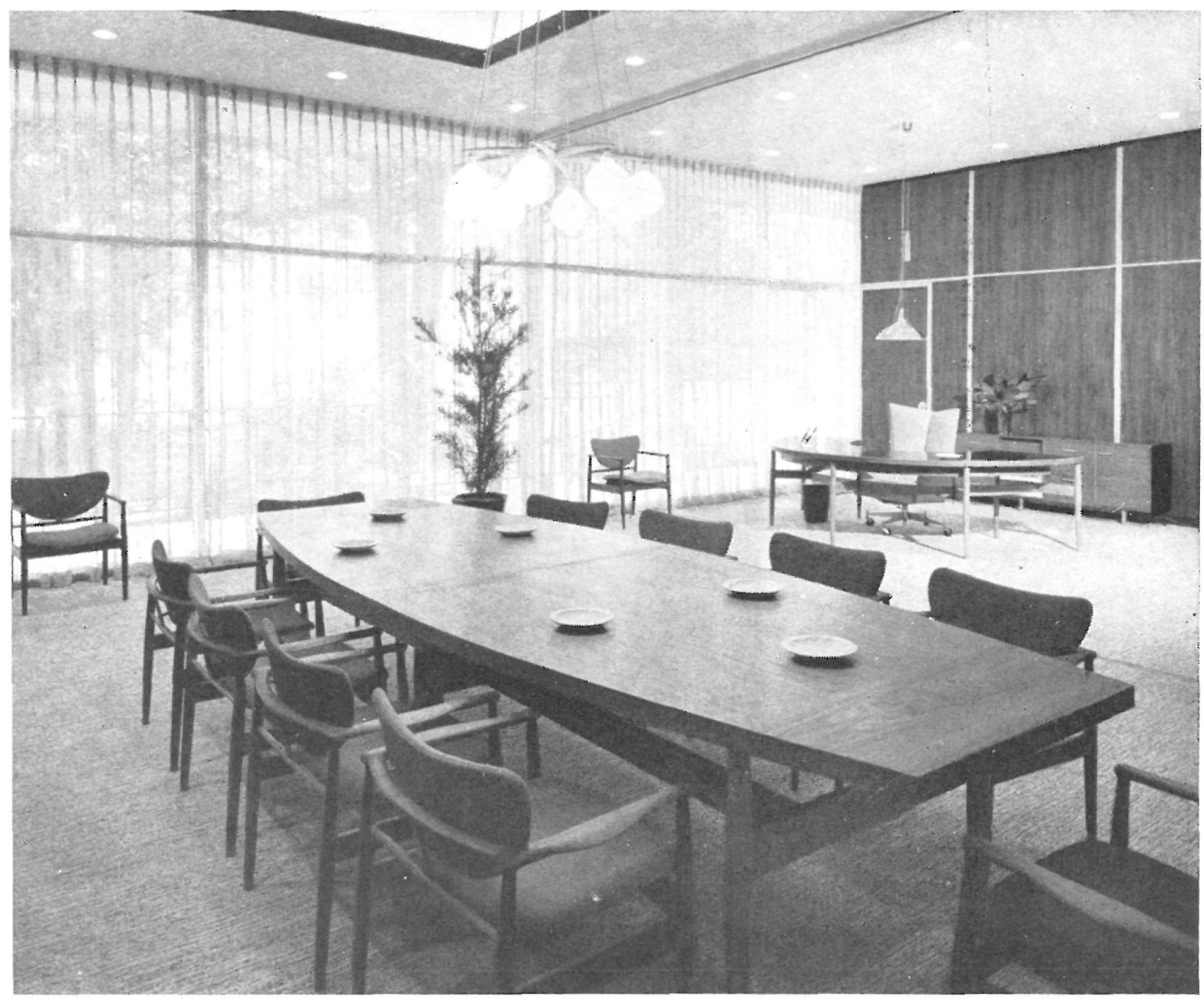

Consta de 132 paneles de hormigón armado formando una integración estética y estructural, sobre los que se representa un bajorrelieve abstracto trabajado en arenisca, y en el que se observan objetos y figuras de la vida ordinaria, incluyendo desde un huevo en una sartén y un grupo familiar alrededor del hogar, hasta la impresión no tan corriente del pie izquierdo de $\mathrm{Ma}$ rilyn Monroe El sol en su recorrido con diario, presta a este bajorrelieve diferentes tonalidades de color y variantes subyugadoras de luces y sombras, infundiéndole caracteres distintos según la hora, la época y el día. El propio Nivola se expresa así: "Se trata de la Escultura integrada perfectamente en la Arquitectura; no sólo de una mera ornamentación superficial y vana, sino de un concepto estructural netamente a escala con la propia Arquitectura."

Las fachadas norte y sur, en toda su altura, están integradas por paneles de cristal sobre carpintería de aluminio, con zonas transparentes y zonas absorbentes del calor y la luz, lo cual permite una iluminación espléndida $e$ inteoral, una difusión confortable y funcional la observación perfecta del panorama desde interior una del panorama desde el interior-y una ultima y muy importante función reflectante, que reproduce la vegetación próxima y el esplendor de los cielos, cada día diferentes.

\section{Interiores:}

El núcleo central alberga todos los servicios de aseo, escaleras, ascensores, sistemas y conductos eléctricos, de aire acondicionado e instalaciones diversas. A su alrededor y en contraste con esta zona bloqueada se desarrollan las oficinas, planeadas con su módulo regulador $1,50 \times 1,50 \mathrm{~m}$, amplias $\mathrm{y}$ libres, de $1,50 \times 1,50 \mathrm{~m}$, amplias y libres, sin tiendo una máxima y variable utilización del espacio disponible.

La planta principal comprende: el vestíbulo, oficinas rectoras, sala de consejos y oficinas generales. Sus fachadas acristaladas, retranqueadas respecto de los elementos resistentes, dejan, entre unas $y$ otros una galería porticada y permite la observación amplísima del permite paisaje circundante. En la planta inferior se incluyen: los almacenes, instalaciones y salas de máquinas, cafetería y algunos despachos.

Toda la obra está realizada con un mimo extraordinario, y en el más ligero detalle se ve la armonía y perfecto equilibrio entre el confort más agradable y el funcionalismo más racional. El saloncito de reposo de empleados, en la parte posterior del edificio, está impregnado de un sabor familiar extrapregnado de un sabor faminamente acogedor.
ordinariamente

Finalmente, el edificio entero se apoya o flota sobre una especie de barcaza de hormigón, anclada con pilotes, lo cual ha evitado la gravosa perforación, hasta profundidades de $40 \mathrm{~m}$, para buscar el firme rocoso, a través de capas menos firmes. 